www.stefancreemers.be $• \bowtie$ info@stefancreemers.be

\title{
Ranking Indices for Mitigating Project Risks
}

\author{
Stefan Creemers \\ Erik Demeulemeester \\ Stijn Van de Vonder
}

Abstract - The goal of project risk management is to provide insight into the risk profile of a project as to facilitate decision makers to mitigate the impact of risks on project objectives such as budget and time. A popular approach to determine where to focus mitigation efforts, is the use of so-called ranking indices. Ranking indices allow the ranking of project activities (or risks) based on the impact they have on project objectives. A distinction needs to be made between activity-based ranking indices (those that rank activities) and risk-driven ranking indices (those that rank risks). Because different ranking indices result in different rankings of activities and risks, one might wonder which ranking index is best? In this article, we provide an answer to this question. Our contribution is threefold: (1) we set up a large computational experiment to assess the efficiency of ranking indices in the mitigation of risks; (2) we develop two new ranking indices that outperform existing ranking indices and (3) we show that a risk-driven approach is more efficient than an activity-based approach.

Keywords - project management, risk management, risk mitigation, ranking index, simulation experiment

\section{Introduction}

Worldwide, projects are still struggling to meet their objectives [12]. During project execution, unforeseen events arise that disrupt plans and budgets and that result in substantial overruns. Risk management is widely recognized as a compulsory discipline to deal with this kind of project uncertainty.

The Project Management Institute [11] defines risk management as the process that deals with the planning, identification, analyzing, responding, 
www.stefancreemers.be $\bowtie$ info@stefancreemers.be

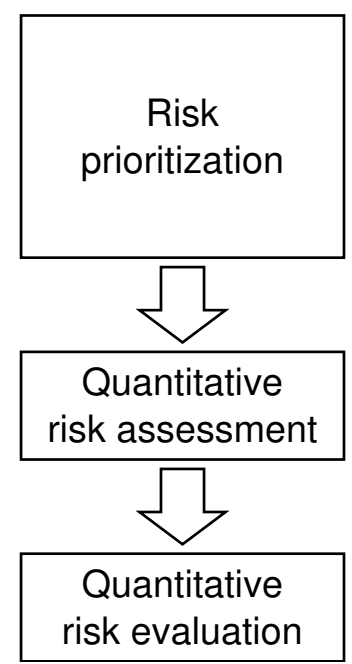

Get ordinal estimates of the probability of
occurrence and the impact of a risk

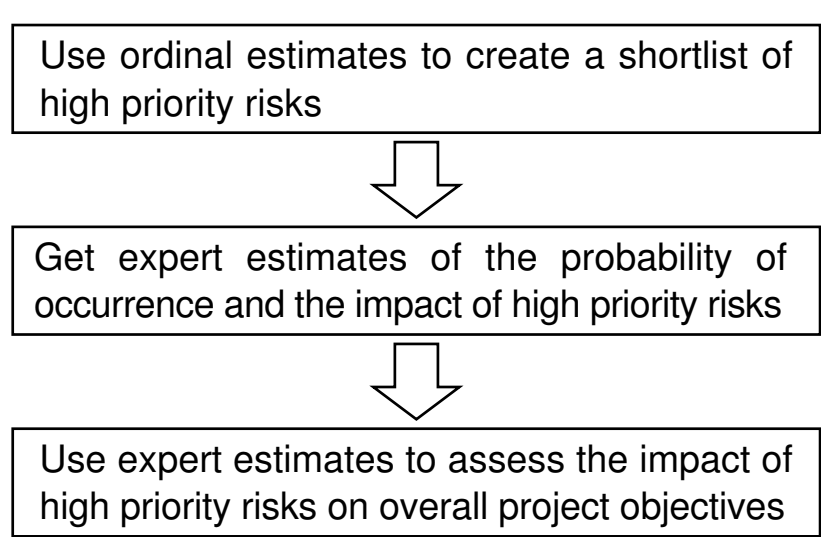

Figure 1: Overview of the risk analysis process

monitoring and controlling of project risks. In this article, we focus on the risk analysis process and its effect on the risk response process. The risk analysis process can be divided into a number of subprocesses: risk prioritization, quantitative risk assessment and quantitative risk evaluation. Risk prioritization is a qualitative procedure that allows to prioritize the risks that were identified in an earlier stage of the risk management process. It requires ordinal estimates of both the probability of occurrence and the impact of a risk. These ordinal estimates are then used to create a shortlist of high priority risks (analogous to the Pareto principle). Further risk analysis efforts should focus on these high priority risks. Quantitative risk assessment is the procedure in which experts provide detailed estimates of the probability of occurrence and the impact of high priority risks. These estimates are used in the quantitative risk evaluation procedure to analyze the impact of the shortlisted risks on overall project objectives. Figure 1 provides a short overview of the dynamics of the risk analysis process.

The goal of risk analysis is to generate insight into the risk profile of a project and to use these insights to drive the risk response process [11]. The insights generated include: the probability of achieving a specific project outcome, the distribution function of the project completion time, etc. The risk response process will use these insights to define practical risk responses 
www.stefancreemers.be $\bowtie$ info@stefancreemers.be

that allow project managers to mitigate risks (i.e. to reduce the impact of risks on project objectives). A popular approach to determine where to focus mitigation efforts is the use of so-called ranking indices. Ranking indices allow the ranking of project activities (or risks) based on the impact they have on project objectives. Different ranking indices may result in different decisions and as such, one might wonder which ranking index is best? It is exactly this question that we will address in this article.

\section{Stochastic project scheduling}

The Critical Path Method (CPM) is developed in the 50's by DuPont Corporation and provides the foundations of modern project scheduling. It represents a project as an activity network which is a graph $G=(N, A)$ that consists of a set of nodes $N=\{1,2, \ldots, n\}$ and a set of $\operatorname{arcs} A=\{(i, j) \mid i, j \in N\}$. The nodes represent project activities whereas the arcs that connect the nodes represent precedence relationships. Activities 1 and $n$ are referred to as the dummy-start and the dummy-end activity and represent the start and the completion of the project respectively. Each activity $j$ has a deterministic activity duration $d_{j}$ and can only start when its predecessors have finished. CPM adopts an early start schedule in which activities are scheduled to start as soon as possible. The early start schedule may be represented by a vector of earliest start times $\mathfrak{s}=\left\{s_{1}, s_{2}, \ldots, s_{n}\right\}$. The earliest start time of an activity $j$ is defined as follows:

$$
s_{j}=\max \left\{f_{i} \mid(i, j) \in A\right\},
$$

where $f_{j}$ is the earliest finish time of an activity $j$ and equals:

$$
f_{j}=s_{j}+d_{j}
$$

By convention, the project starts at time instance 0 (i.e. $s_{1}=0$ ). According to $\mathrm{CPM}$, the project completion time $c$ is computed as follows:

$$
c=f_{n}
$$

The longest path of the scheduled activities is called the critical path and the activities on this path are critical activities.

Since the establishment of CPM, many extensions of the basic model have been introduced: generalized precedence relationships, resource-constrained 
www.stefancreemers.be $• \bowtie$ info@stefancreemers.be

project scheduling, multi-mode scheduling, critical chain buffer management, etc. We refer to Demeulemeester and Herroelen [4] for an extensive overview of the field. In this article, we are particularly interested in what is called stochastic project scheduling or stochastic CPM. Stochastic CPM acknowledges that activity durations are not deterministic. We model the duration of an activity $j$ as a positive random variable $D_{j}$. Because the duration of an activity is a random variable, the earliest start and finish times of an activity are random variables as well. Let $S_{j}$ and $F_{j}$ denote the random variable of the earliest start and finish times of an activity $j$ respectively. The project completion time is a random variable $C$ which is a function of $D_{j}$. Calculating the distribution function of $C$ is proven to be \#P-complete [7] and thus requires approximative methods such as Monte Carlo simulation [14]. Monte Carlo simulation is used to virtually execute a project a large number of times, providing insight and allowing the project manager to enhance the actual execution of the project.

We will use Monte Carlo simulation to generate random variates of $D_{j}$. Let $\mathbf{d}_{j}=\left\{d_{j, 1}, d_{j, 2}, \ldots, d_{j, q}\right\}$ denote the vector of $q$ random variates of $D_{j}$ (where $q$ represents the number of simulation iterations). We refer to $\mathbf{d}_{j}$ as the vector of realized durations of $D_{j}$. In addition, define $\mathbf{s}_{j}$ the vector of realized earliest start times of an activity $j$ :

$$
\mathbf{s}_{j}=\max \left\{\mathbf{f}_{i} \mid(i, j) \in A\right\},
$$

where $\mathbf{f}_{j}$ is the vector of realized earliest finish times of an activity $j$ and equals:

$$
\mathbf{f}_{j}=\mathbf{s}_{j}+\mathbf{d}_{j} .
$$

The vector of realized project completion times $\mathbf{c}$ is defined as follows:

$$
\mathbf{c}=\mathbf{f}_{n} .
$$

It is clear that $\mathbf{s}_{j}, \mathbf{f}_{j}$ and $\mathbf{c}$ are vectors of random variates of random variables $S_{j}, F_{j}$ and $C$ respectively.

\section{Towards a risk-driven approach}

One of the main challenges in project risk management is to estimate and to model the uncertainty of activity durations. Often, it is assumed that the duration of an activity follows a distribution that captures all uncertainty that 
www.stefancreemers.be $• \bowtie$ info@stefancreemers.be

originates from the occurrence of risks (popular distributions include: the triangular distribution, the beta distribution and the normal distribution). As such, risk assessment boils down to providing estimates of activity duration distribution parameters. We refer to this approach as the activity-based approach.

In this article we argue that the activity-based approach is inherently flawed. As Hulett [8] points out, there is no clear link between the impact of identified risks on the duration of an activity and the distribution of the activity duration itself (i.e. the activity-based approach is unable to identify the root causes of the uncertainty in the duration of an activity). In addition, our experience learns that practitioners have a hard time assessing uncertainty by estimating the parameters of an activity duration distribution.

To resolve the problems of the activity-based approach, we devise a riskdriven approach in which the impact of each risk is assessed individually and is mapped to the duration of an activity afterwards. Our approach is based on previous work by Schatteman et al. [10] and Van de Vonder [13] and is similar to the risk-driver approach of Hulett [8]. Contrary to the activity-based approach, we focus on risks as primary sources of uncertainty. In what follows, we adopt an integrated approach that relies on Monte Carlo simulation to evaluate the impact of risks on activity durations and on the project completion time. Figure 2 presents a visual overview from which it is clear that a risk-driven approach assesses the impact of root risks on the uncertainty of the activities and on the project completion time. An activity-based approach on the other hand, assesses only the uncertainty of the activities without observing the root risks that cause this uncertainty.

To further support the risk-driven approach, we provide the following example. Consider an activity whose duration is impacted by two risks. The first risk has a small impact yet a large probability of occurrence whereas the second risk has a large impact but a small probability of occurrence. The resulting probability distribution of the duration of the activity is presented in Figure 3. In the figure, the dotted line represents the best fit of the triangular distribution (we use the triangular distribution because it is very popular in practice as it allows to assess a worst case, best case and most plausible case scenario). It is clear that fitting a triangular distribution (or any other would result in significant errors. In addition, it would be very hard for practitioners to estimate the parameters of the fitted distribution. Assessing the probability of occurrence and the impact of both risks on the other hand, would be a manageable task and would result in the correct 
Activity-based approach

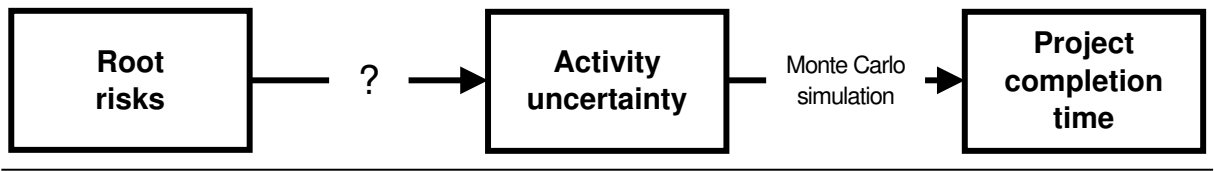

Risk-driven approach

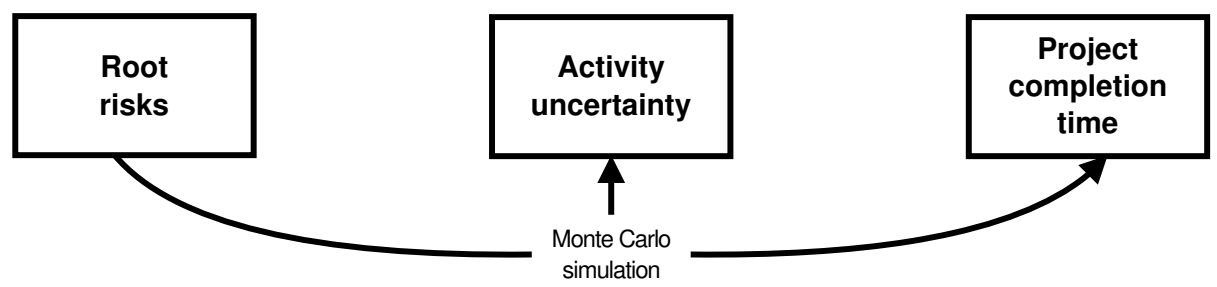

Figure 2: Activity-based versus risk-driven approach

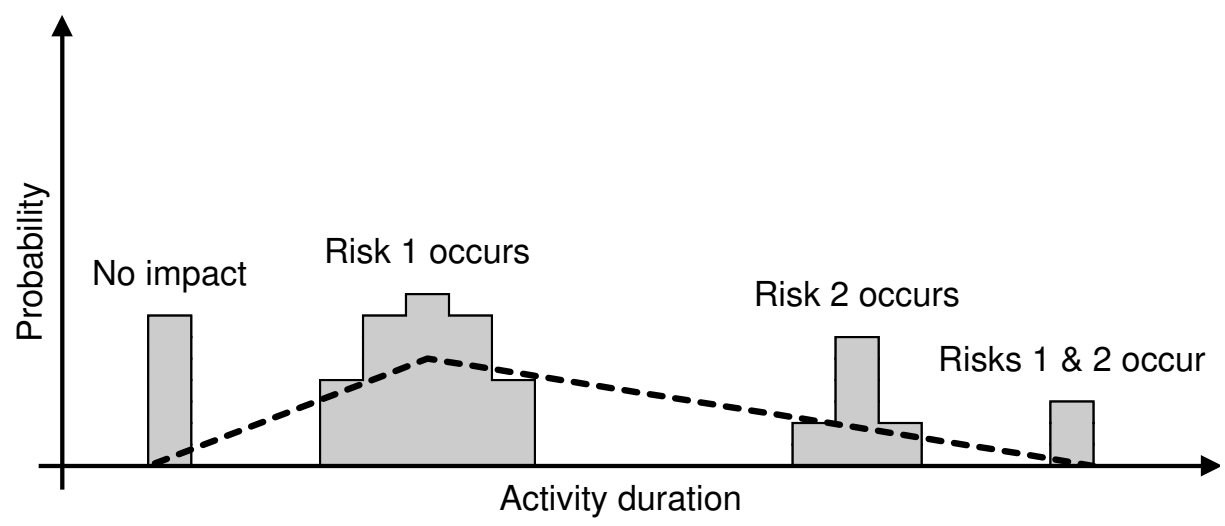

Figure 3: Example distribution of the duration of an activity 
www.stefancreemers.be $\bowtie$ info@stefancreemers.be

distribution of the duration of the activity.

In order to formally define risks and their impacts, let $R=\{1,2, \ldots, r\}$ denote the set of risks and let $\mathbf{M}=\left\{M_{j, e} \mid j \in N \wedge e \in R\right\}$ denote the set of risk impacts, where $M_{j, e}$ is the random variable of the risk impact of a risk $e$ on the duration of an activity $j$. Let $\mathbf{m}_{j, e}$ represent the vector of random variates of $M_{j, e}$ and define $\mathbf{d}_{j}^{(E)}=\left\{d_{j, 1}^{(E)}, d_{j, 2}^{(E)}, \ldots, d_{j, q}^{(E)}\right\}$, the vector of random variates of the duration of an activity $j$ subject to a set of risks $E \subseteq R$ :

$$
\mathbf{d}_{j}^{(E)}=d_{j}+\sum_{e \in E} \mathbf{m}_{j, e}
$$

where $d_{j}$ is the deterministic (i.e. risk-free) duration of an activity $j$. From $\mathbf{d}_{j}^{(E)}$ we obtain $\mathbf{s}_{j}^{(E)}=\left\{s_{j, 1}^{(E)}, s_{j, 2}^{(E)}, \ldots, s_{j, q}^{(E)}\right\}, \mathbf{f}_{j}^{(E)}=\left\{f_{j, 1}^{(E)}, f_{j, 2}^{(E)}, \ldots, f_{j, q}^{(E)}\right\}$ and $\mathbf{c}^{(E)}=\left\{c_{1}^{(E)}, c_{2}^{(E)}, \ldots, c_{q}^{(E)}\right\}$ by generalizing Equations 4,5 and 6 .

$$
\begin{aligned}
\mathbf{s}_{j}^{(E)} & =\max \left\{\mathbf{f}_{i}^{(E)} \mid(i, j) \in A\right\} \\
\mathbf{f}_{j}^{(E)} & =\mathbf{s}_{j}^{(E)}+\mathbf{d}_{j}^{(E)} \\
\mathbf{c}^{(E)} & =\mathbf{f}_{n}^{(E)}
\end{aligned}
$$

The expected project delay over $q$ simulation iterations is defined as follows:

$$
\Delta^{(E)}=\frac{1}{q} \sum_{p=1}^{q} c_{p}^{(E)}-c,
$$

where $c$ is the risk-free project completion time and is computed using Equation 3 .

\section{Effective risk mitigation}

Most commercial risk analysis software packages provide the functionality to generate insight into the source of project overruns. The activities (or the risks) that contribute most to the project overrun are identified using ranking indices. Let $(\cdot)_{j}^{(E)}$ and $(\cdot)_{e}^{(E)}$ denote the ranking values of a ranking index $(\cdot)$ for an activity $j$ and a risk $e$ when activity durations are subject to a set of risks $E$. The larger the ranking value, the larger the contribution 
www.stefancreemers.be $\bullet$ info@stefancreemers.be

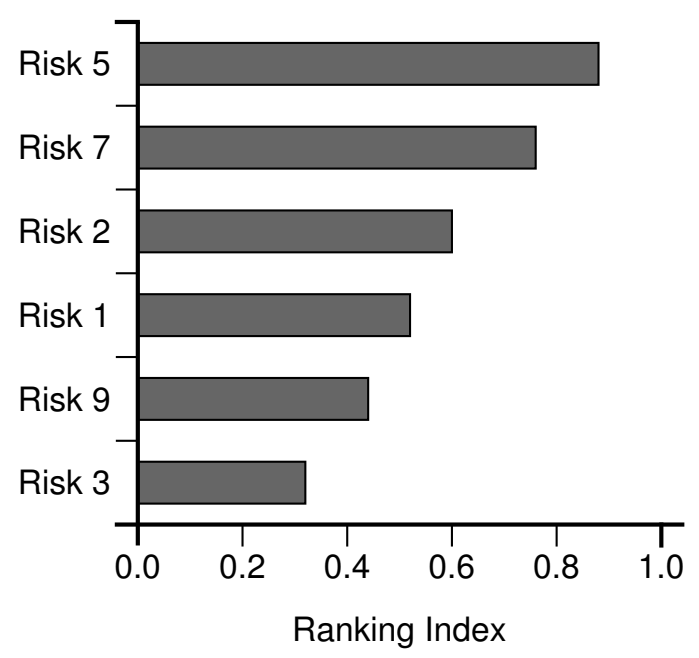

Figure 4: Tornado graph

of the activity (or the risk) to the project overrun. The ranking of activities (or risks) is typically visualized using a tornado graph (see Figure 4 for an example of a tornado graph).

In the remainder of this Section, we will first provide an overview of the existing ranking indices. Next, we will introduce two new ranking indices that will be compared with the existing ones in a computational experiment.

\subsection{Literature review}

In this Section, we provide an overview of the existing ranking indices to determine the contribution of activities (or risks) to the project overrun. We refer to Elmaghraby [5] and Demeulemeester and Herroelen [4] for a more detailed discussion on the ranking indices discussed below.

\subsubsection{Critical Activities ( $C A)$}

A common practice in project risk management is to focus mitigation efforts on the critical activities of the deterministic early start schedule $\mathfrak{s}[6]$. The Critical Activities $(C A)$ ranking values are computed as follows:

$$
\mathrm{CA}_{j}^{(E)}=\delta_{j}
$$


www.stefancreemers.be $\bullet$ info@stefancreemers.be

where $\delta_{j}$ equals 1 if $j$ is critical in $\mathfrak{s}$ and 0 otherwise.

While easy to implement, $C A$ does not recognize the uncertain nature of a project. In addition, all activities on the critical chain have an equal ranking value, thereby severely limiting the discriminative power of the ranking index.

\subsubsection{Activity Criticality Index $(A C I)$}

In stochastic CPM, the critical path is not fixed. For instance, the occurrence of risks may alter the critical path in a given network. The Activity Criticality Index $(A C I)$ recognizes that almost any path and any activity can become critical with a certain probability [14]. When using Monte Carlo simulation, the $A C I$ of an activity is simply the proportion of simulation iterations in which the activity was critical:

$$
\mathrm{ACI}_{j}^{(E)}=\frac{1}{q} \sum_{p=1}^{q} \delta_{j, p}^{(E)},
$$

where $\delta_{j, p}^{(E)}$ equals 1 if $j$ is critical in $\mathfrak{s}_{p}^{(E)}$ and 0 otherwise; $\mathfrak{s}_{p}^{(E)}$ is the early start schedule during a simulation iteration $p$ when activity durations are subject to a set of risks $E$.

\subsubsection{Significance Index $(S I)$}

The Significance Index $(S I)$ was developed by Williams [16] as an answer to criticism on $A C I$. When using Monte Carlo simulation, $S I$ is computed as follows:

$$
\begin{aligned}
\mathrm{SI}_{j}^{(E)} & =\frac{1}{q}\left(\frac{1}{\sum_{p=1}^{q} c_{p}^{(E)}}\right)\left[\sum_{p=1}^{q}\left(\frac{d_{j, p}^{(E)}}{d_{j, p}^{(E)}+\mathrm{TF}_{j, p}^{(E)}} c_{p}^{(E)}\right)\right], \\
& =E\left[\frac{\mathbf{d}_{j}^{(E)}}{\mathbf{c}^{(E)} \mathbf{d}_{j}^{(E)} \mathbf{T} \mathbf{F}_{j}^{(E)}}\right],
\end{aligned}
$$

where $\mathrm{TF}_{j, p}^{(E)}$ is the total float of an activity $j$ during a simulation iteration $p$ when activity durations are subject to a set of risks $E$ and $\mathbf{T F}_{j}^{(E)}=$ $\left\{\mathrm{TF}_{j, 1}^{(E)}, \mathrm{TF}_{j, 2}^{(E)}, \ldots, \mathrm{TF}_{j, q}^{(E)}\right\}$ (refer to Demeulemeester and Herroelen [4] for a definition of total float). 
www.stefancreemers.be $\bowtie$ info@stefancreemers.be

\subsubsection{Cruciality Index $(C R I)$}

The cruciality index $(C R I)$ is defined as the absolute value of the correlation between the duration of an activity and the total project duration. When using Monte Carlo simulation, $C R I$ is computed as follows:

$$
\mathrm{CRI}_{j}^{(E)}=\left|\operatorname{corr}\left(\mathbf{d}_{j}^{(E)}, \mathbf{c}^{(E)}\right)\right| \text {. }
$$

\subsubsection{Spearman Rank Correlation ( $S R C)$}

Cho and Yum [1] have criticized CRI because it assumes a linear relationship between the duration of an activity and the project completion time. They propose the use of a non-linear correlation measure such as the Spearman rank correlation coefficient. The Spearman Rank Correlation index $(S R C)$ is computed as follows:

$$
\mathrm{SRC}_{j}^{(E)}=\left|\operatorname{corr}\left(\operatorname{rank}\left(\mathbf{d}_{j}^{(E)}\right), \operatorname{rank}\left(\mathbf{c}^{(E)}\right)\right)\right| .
$$

\subsubsection{Schedule Sensitivity Index $(S S I)$}

The PMI Body of Knowledge [11] and Vanhoucke [15] define a ranking index that combines $A C I$ and the variance of $\mathbf{d}_{j}^{(E)}$ and $\mathbf{c}^{(E)}$. When using Monte Carlo simulation, $S S I$ is computed as follows:

$$
\mathrm{SSI}_{j}^{(E)}=\mathrm{ACI}^{(E)} \sqrt{\frac{\operatorname{Var}\left(\mathbf{d}_{j}^{(E)}\right)}{\operatorname{Var}\left(\mathbf{c}^{(E)}\right)}} .
$$

\subsubsection{Risk-Driven Ranking Indices}

All prior ranking indices have been criticized in the literature (refer to Williams [16], Elmaghraby [5] and Cui et al. [2]) and are primarily designed to rank activities, not risks. In this (and later) Sections we will introduce risk-driven ranking indices.

To the best of our knowledge, Hulett [8] is the only reference that explicitly refers to a risk-driven ranking index. He proposes a simple adaptation of the CRI such that it calculates the absolute correlation between the impact of a risk and the project completion time. When using Monte Carlo Simulation, the Cruciality Index for Risks $(C R I R)$ is computed as follows:

$$
\operatorname{CRIR}_{e}^{(E)}=\left|\operatorname{corr}\left(\mathbf{m}_{e}, \mathbf{c}^{(E)}\right)\right|
$$


www.stefancreemers.be $\bullet$ info@stefancreemers.be

where $\left(\mathbf{m}_{e}=\sum_{j \in N} \mathbf{m}_{e, j}\right)$ and $e \in E$. A similar adaptation may be made with respect to $S R C$ :

$$
\operatorname{SRCR}_{e}^{(E)}=\left|\operatorname{corr}\left(\operatorname{rank}\left(\mathbf{m}_{e}\right), \operatorname{rank}\left(\mathbf{c}^{(E)}\right)\right)\right| .
$$

No simple risk-driven adaptation exists for the other activity-based ranking indices (i.e. $C A, A C I, S I$ and $S S I$ ).

\subsection{Two new ranking indices}

The aim of the new ranking indices is to redistribute the project delay over the combinations of activities and risks that cause the delay. More formally, the Critical Delay Contribution (CDC) of an activity $j$ and a risk $e$ may be expressed as follows:

$$
\begin{aligned}
\mathrm{CDC}_{j, e}^{(E)} & =\frac{1}{q} \frac{\sum_{p=1}^{q} m_{j, e, p} \delta_{j, p}^{(E)}\left(c_{p}^{(E)}-c\right)}{\sum_{j \in N} \sum_{e \in E} \sum_{p=1}^{q} m_{j, e, p} \delta_{j, p}^{(E)}}, \\
& =E\left[\frac{\mathbf{m}_{j, e} \mathbf{y}_{j}^{(E)}}{\sum_{j \in N} \sum_{e \in E} \mathbf{m}_{j, e} \mathbf{y}_{j}^{(E)}}\right] \Delta^{(E)},
\end{aligned}
$$

where $\mathbf{y}_{j}^{(E)}=\left\{\delta_{j, 1}^{(E)}, \delta_{j, 2}^{(E)}, \ldots, \delta_{j, q}^{(E)}\right\}$.

From $\mathrm{CDC}_{j, e}^{(E)}$ it is easy to obtain both an activity-based as well as a risk-driven ranking index:

$$
\begin{aligned}
\operatorname{CDCR}_{e}^{(E)} & =\sum_{j \in N} \operatorname{CDC}_{j, e}^{(E)}, \\
\mathrm{CDCA}_{j}^{(E)} & =\sum_{e \in E} \mathrm{CDC}_{j, e}^{(E)} .
\end{aligned}
$$

$C D C A$ and $C D C R$ are both new ranking indices whose dynamics are best explained using an example. Consider the project presented in Figure 5. The project has three non-dummy activities (i.e. the start and the completion of the project are represented by activity 1 and 5 respectively). The risk-free activity durations are $\left(d_{2}=2\right) ;\left(d_{3}=3\right)$ and $\left(d_{4}=6\right)$. Precedence relations 


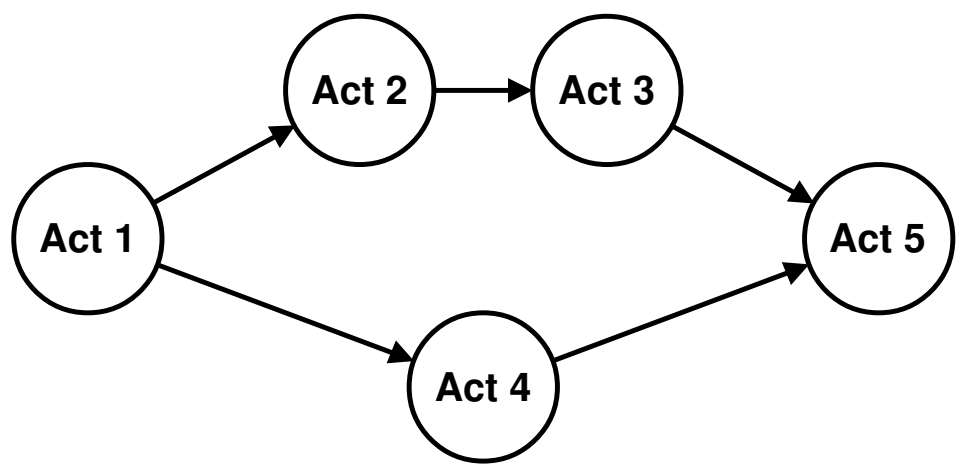

Figure 5: Example project network

\begin{tabular}{|lc|ccc|c|}
\hline Act & Risk & \multicolumn{4}{|c|}{$m_{j, e, p}$} \\
& & $p=1$ & $p=2$ & $p=3$ & CDC \\
\hline$j=2$ & $e=1$ & $+1(0)$ & $+1(0.66)$ & $+1(0.75)$ & $\mathrm{CDC}_{2,1}^{(E)}=0.47$ \\
& $e=2$ & - & $+2(1.33)$ & - & $\mathrm{CDC}_{2,2}^{(E)}=0.44$ \\
$j=3$ & $e=3$ & - & - & $+3(2.25)$ & $\mathrm{CDC}_{3,3}^{(E)}=0.75$ \\
$j=4$ & $e=1$ & $+1(1)$ & $+1(0)$ & $+1(0)$ & $\mathrm{CDC}_{4,1}^{(E)}=0.33$ \\
\hline$c_{p}^{(E)}$ & & 7 & 8 & 9 & $E\left[\mathbf{c}^{(E)}\right]=8$ \\
$c_{p}^{(E)}-c$ & & 1 & 2 & 3 & $\Delta^{(E)}=2$ \\
\hline
\end{tabular}

Table 1: Computing the CDC

(finish-start) exist between activities 1 and 2, activities 1 and 4 , activities 2 and 3, activities 3 and 5 and activities 4 and 5 . The risk-free project completion time is $(c=6)$. Three risks have been identified and their respective risk impacts are presented in Table 1. For the sake of simplicity, we consider only three simulation iterations (i.e. $q$ equals 3 ).

For example, we observe that activities 2 and 3 are critical during simulation iteration $(p=2)$ and that the critical path has a length of eight time units, resulting in a project delay of two time units (i.e. $c_{p}^{(E)}-c=8-6=2$ ). As a consequence, during simulation iteration $(p=2)$, the CDC of risk 1 on activity 2 is indicated between brackets and equals $\left(\frac{1(8-6)}{1+2}=2 / 3\right)$.

Table 2 illustrates how the numbers in Table 1 may be aggregated both at the level of each activity as well as at the level of each risk, resulting in 
www.stefancreemers.be $\bowtie$ info@stefancreemers.be

\begin{tabular}{|c|ccc|c|}
\hline & Risk 1 & Risk 2 & Risk 3 & CDCA $_{j}^{(E)}$ \\
\hline Act 2 & 0.47 & 0.45 & - & 0.92 \\
Act 3 & - & - & 0.75 & 0.75 \\
Act 4 & 0.33 & - & - & 0.33 \\
\hline $\mathrm{CDCR}_{e}^{(E)}$ & 0.8 & 0.45 & 0.75 & $\Delta^{(E)}=2$ \\
\hline
\end{tabular}

Table 2: Aggregation of CDC

ranking values $\mathrm{CDCA}_{j}^{(E)}$ and $\mathrm{CDCR}_{e}^{(E)}$ respectively.

The performance of both $C D C A$ and $C D C R$ will be evaluated in the upcoming Section.

\section{Computational Experiment}

The goal of the computational experiment is to evaluate the resilience of ranking indices in a wide variety of settings, using an extensive experimental design. At the core of our experimental design are the PSPLIB J120 project networks (refer to Kolisch and Sprecher [9] for a discussion of this set of project networks). In our computational experiment we take into account five parameters to characterize risks: (1) risk uniformity; (2) risk quantity; (3) risk probability; (4) risk impact and (5) risk correlation. The settings of these parameters are based on our experience in the risk management field and combine to 48 distinct risk profiles that are to be evaluated. The details of the computational experiments may be found in Creemers et al. [3].

We test the mitigation potential of each ranking index using a stepwise procedure. In each step, the selected ranking index is used to identify the risk that contributes most to the delay of the project. Next, this risk is eliminated (i.e. is fully mitigated). After mitigation, we rerun the simulation and recalculate the expected project delay. Once more, the selected ranking index is used to identify and to mitigate the risk that has the largest impact on the project delay. This process continues until 10 risks have been mitigated. More formally, let $E(\cdot)_{x}$ denote the set of risks after mitigation of $x$ risks using ranking index $(\cdot)$, with $\left(E(\cdot)_{0}=R\right),\left(E(\cdot)_{r}=\emptyset\right)$ and $x \in\{0,1, \ldots, 10\}$.

In our experiment, we evaluate a total of 12 ranking indices. The ten ranking indices discussed in Section 4 ( $C A, A C I, S I, C R I, S R C, S S I, C R I R$, $S R C R, C D C A$ and $C D C R$ ) as well as two additional ranking indices: (1) 
www.stefancreemers.be $\bullet$ info@stefancreemers.be

$R A N D$ randomly selects a risk from those risks still active and may be considered as a worst-case scenario and (2) OPT is a greedy optimal ranking index that evaluates the elimination of all risks after each simulation run and selects the best risk to mitigate. OPT may be considered as a best-case scenario but has limited practical value due to its computational requirements.

With respect to the activity-based ranking indices, selecting the largest risk is a two-step procedure. In a first step, the highest-ranked activity is selected. In a second step, the risk that has the largest expected impact on the selected activity is identified as the highest-ranked risk.

Figure 6 gives an overview of the average performance of the activitybased ranking indices with respect to measure $\operatorname{RRD}^{\left(E()_{x}\right)}$ for the range starting from $(x=0)$ until $(x=10)$ (i.e. ten risks have been mitigated). The data are aggregated over all 600 project networks in the PSPLIB J120 data sets and over all 48 risk profiles. We observe that the mitigation of risks results in a decrease of the expected project delay for each ranking index. Because $R A N D$ randomly selects risks, its improvement is linear with the number of risks mitigated. All other ranking indices follow a convex function, implying that risks with a larger impact on the project delay are selected first. One might conclude that $C D C A$ (the activity-based ranking index proposed in this article) outperforms all other activity-based ranking indices. However, it is clear that there still exists a gap between the performance of the activity-based indices and the OPT procedure.

Similarly to Figure 6, Figure 7 presents the performance of risk-driven ranking indices with respect to measure $\operatorname{RRD}^{\left(E(\cdot)_{x}\right)}$. We observe that $C R I R$ and $S R C R$ have similar performance and are able to outperform the activitybased ranking indices. Of larger importance, however, is the observation that $C D C R$ (the risk-driven ranking index proposed in this article) easily outperforms $C R I R$ and $S R C R$ and even matches the performance of the $O P T$ procedure. It is clear that $C D C R$ sets a new standard in the field of ranking indices.

\section{Conclusion}

In this article we introduced a quantitative, new approach to project risk analysis that allows to address the risk response process in a scientificallysound manner. We have shown that a risk-driven approach is more efficient than an activity-based approach when it comes to analyzing risks. Therefore, 
www.stefancreemers.be $\bowtie$ info@stefancreemers.be

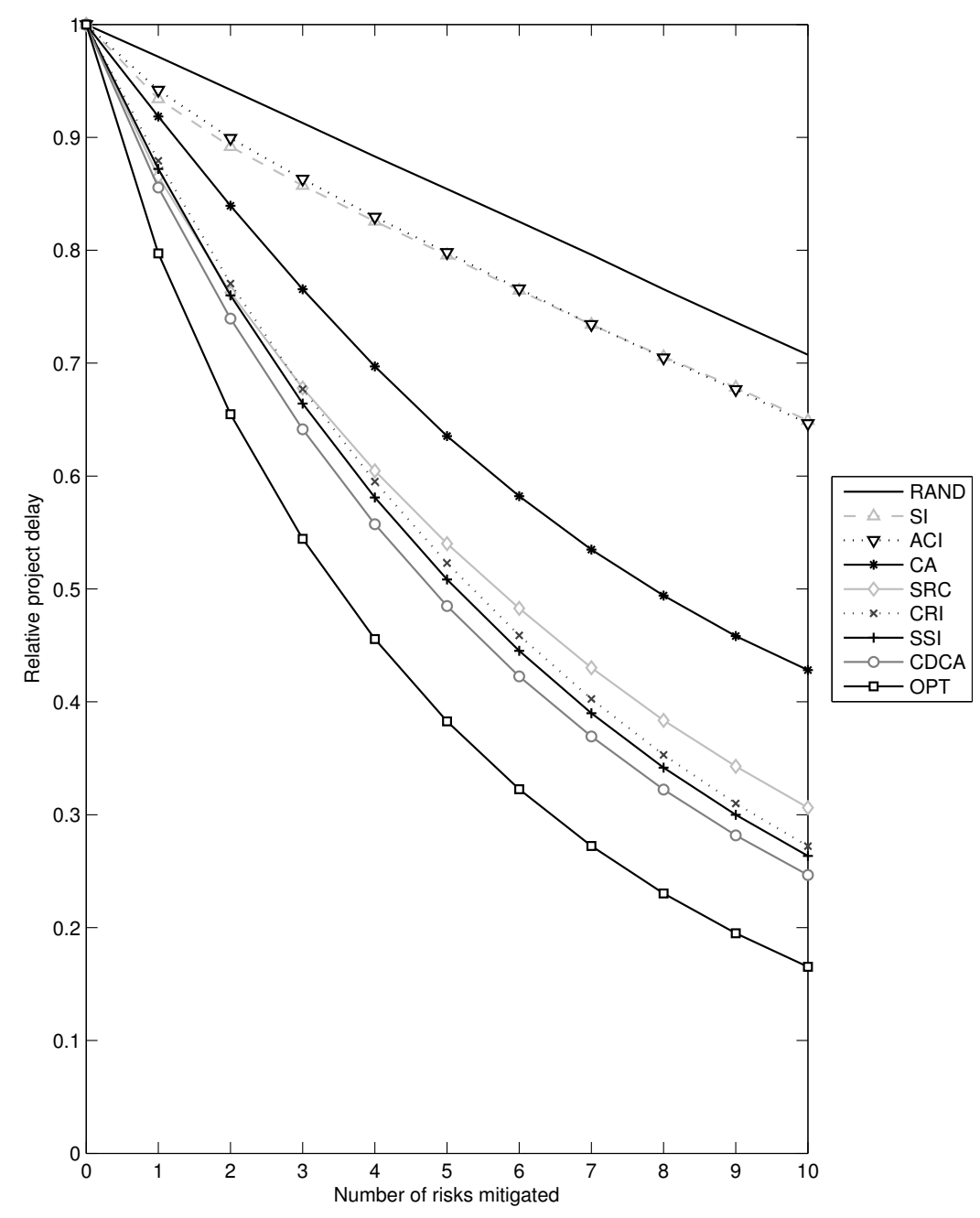

Figure 6: Mitigation potential of activity-based ranking indices 
www.stefancreemers.be $\bowtie$ info@stefancreemers.be

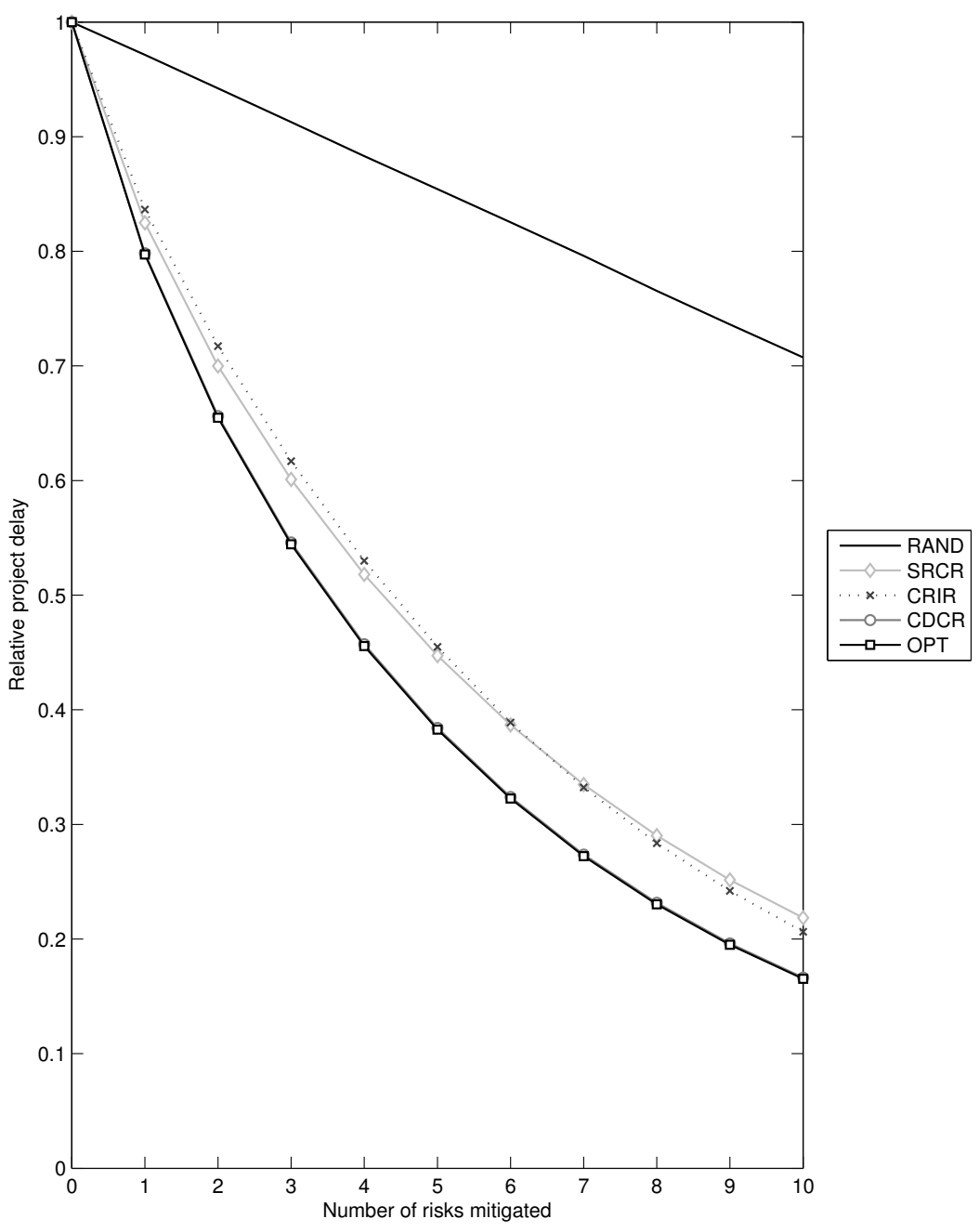

Figure 7: Mitigation potential of risk-driven ranking indices 
www.stefancreemers.be $\bowtie$ info@stefancreemers.be

project risk management should focus on assessing the uncertainty caused by risks themselves (i.e. the root cause) rather than evaluating the uncertainty at the level of activities.

In addition, we developed two new ranking indices to assist project managers in determining where to focus their risk mitigation efforts. Ranking indices allow to identify the activities (or risks) that contribute most to the delay of a project. We developed both an activity-based ranking index (that ranks activities) and a risk-driven ranking index (that ranks risks). We refer to these ranking indices as $C D C A$ and $C D C R$ respectively. Both ranking indices outperform existing ranking indices, with $C D C R$ nearly matching the performance of a greedy-optimal procedure. $C D C R$ sets a new standard in the field of ranking indices.

Our conclusions are supported by an extensive simulation experiment and were proven to be robust for a broad range of parameter settings. The contributions of this article may be summarized as follows: (1) we assess the performance of a wide variety of ranking indices using a large simulation experiment; (2) we develop two new ranking indices that outperform existing ranking indices and (3) we show that risk analysis should be risk-driven rather than activity-based.

\section{References}

[1] J.G. Cho, and B.J. Yum, "An uncertainty importance measure of activities in PERT networks," International Journal of Production Research, vol. 35, no. 10, pp. 2737-2758, 1997.

[2] W. Cui, Q. Jiajun, and Y. Chaoyuan, "Criticality measurement in PERT networks," presented at the IEEE International Conference on Systems, Man, and Cybernetics, Taipei, Taiwan, 2006.

[3] S. Creemers, E. Demeulemeester, and S. Van de Vonder, "A new approach for quantitative risk analysis," Department of Decision Sciences and Information Management, FBE, KULeuven, Leuven, Belgium, Technical Report 1029, 2010.

[4] E. Demeulemeester and W. Herroelen, Project scheduling: A research handbook. Boston: Kluwer Academic Publishers, 2002. 
www.stefancreemers.be $\bullet$ info@stefancreemers.be

[5] S. Elmaghraby, "On criticality and sensitivity in activity networks," European Journal of Operational Research, vol. 127, no. 2, pp. 220-238, 2000.

[6] E.M. Goldratt, Critical chain. Great Barrington: The North River Press Publishing Corporation, 1997.

[7] J.N. Hagstrom, "Computational complexity of PERT problems," Networks, vol. 18, no. 2, pp. 139-147, 1988.

[8] D.T. Hulett, Practical Schedule Risk Analysis. England: Gower Publishing Limited, 2009.

[9] R. Kolisch, and A. Sprecher, "PSPLIB - A project scheduling problem library," European Journal of Operational Research, vol. 96, pp. 205-216, 1996.

[10] D. Schatteman, W. Herroelen, A. Boone, and S. Van de Vonder, "A methodology for integrated risk management and proactive scheduling of construction projects," Journal of Construction Engineering $\&$ Management, vol. 134, no. 11, pp. 885-895, 2008.

[11] The Project Management Institute, Guide to the project management body of knowledge (PMBOK guide). Newton Square: The Project Management Institute, 2008.

[12] The Standish Group, Chaos report. Boston: The Standish Group, 2009.

[13] S. Van de Vonder, Proactive-reactive procedures for robust project scheduling. PhD Disseration. Leuven: K.U.Leuven, 2006.

[14] R.M. Van Slyke, "Monte Carlo methods and the PERT problem," Operations Research, vol. 11, no. 5, pp. 839-860, 1963.

[15] M. Vanhoucke, "Using activity sensitivity and network topology information to monitor project time performance," Omega, vol. 38, no. 5, pp. 359-370, 2010.

[16] T.M. Williams, "Criticality in stochastic networks," The Journal of the Operational Research Society, vol. 43, no. 4, pp. 353-357, 1992. 